\title{
Berriane Mohamed, Marocains de l'extérieur 2013
}

\section{Chadia Arab}

\section{(2) OpenEdition \\ 1 Journals}

Édition électronique

URL : https://journals.openedition.org/remi/7519

DOI : 10.4000/remi.7519

ISSN : $1777-5418$

Éditeur

Université de Poitiers

\section{Édition imprimée}

Date de publication : 1 décembre 2015

Pagination : 341-345

ISBN : 979-10-90426-26-9

ISSN : 0765-0752

Référence électronique

Chadia Arab, "Berriane Mohamed, Marocains de l'extérieur 2013 », Revue européenne des migrations

internationales [En ligne], vol. $31-\mathrm{n}^{\circ} 3$ et 4 | 2015, mis en ligne le 01 décembre 2015, consulté le 14 avril 2022. URL : http://journals.openedition.org/remi/7519; DOI : https://doi.org/10.4000/remi.7519 
Berriane, Mohamed

Marocains de l'extérieur 2013. - Rabat:

Fondation Hassan II pour les Marocains

Résidants à l'Étranger, 2014. - 602 p.

ISBN : 978-9954-400-35-7

Cette troisième édition innove grâce à une première partie originale et pertinente qui analyse les thématiques transversales de ces communautés marocaines et pose les jalons des tendances de la recherche actuelle sur les migrations marocaines.

Le premier chapitre présente l'état de la recherche sur les migrations marocaines (Mohamed Berriane et Mohammed Aderghal). Sont mises en exergue la question du retour dans les années 1990, et tout particulièrement la dichotomie entre enracinement dans le pays d'accueil et relation avec le pays d'origine, ainsi que la recomposition des territoires de la mobilité avec une évolution conceptuelle. Le terme de circulation remplace progressivement celui de migration et celle-ci se voit qualifiée de transnationale. Sont aussi évoquées les femmes marocaines qui partent seules, la relation entre la migration et le développement, les nouvelles fonctions migratoires avec l'arrivée des Subsahariens, des Européens et des Asiatiques et de nouveaux concepts tels que la polyspatialité, la migration de bien-être ou encore la résidence touristique de longue durée.

Le deuxième chapitre développe la théorie des transitions migratoires. Hein de Haas remet en cause le schéma explicatif linéaire du push and pull. II conteste la linéarité entre migration et développement. La migration requiert des capacités considérables et le désir de migrer est augmenté par l'accroissement du taux de scolarisation, le contact avec les médias et la prise de conscience des opportunités à l'étranger. Ainsi, on comprend mieux, au Maroc, la relation entre le développement et la persistance d'une émigration ; des mutations récentes apparaissent avec l'immigration subsaharienne.

Le troisième chapitre analyse le rôle des associations de migrants dans le développement du Maroc. L'ONG Migration et Développement devient un acteur incontournable dans la zone Sud du Maroc avec des projets d'électrification des villages, d'éducation informelle, d'équipements hydrauliques, des opérations de construction de route et de centres de santé. Thomas Lacroix interroge le paradoxe entre l'intégration, le renforcement des relations transnationales et l'invention d'une nouvelle citoyenneté détachée des cadres territoriaux.

Au sein du quatrième chapitre, Myriam Cherti propose l'étude de la migration de retour. Cette chercheuse explique que la tendance est à l'augmentation du retour des immigrés en raison de la hausse du chômage et de la crise, de leur faible intégration dans les pays d'accueil, des nouvelles opportunités dans leurs pays d'origine, du contrôle renforcé aux frontières et des politiques migratoires qui affectent les immigrés en situation irrégulière. Des mesures sont développées par le Maroc pour soutenir ces migrants qui reviennent, mais elles sont fragmentaires et se focalisent sur le soutien des émigrés qui souhaitent investir dans leur pays.

Les deux chapitres suivants s'appuient sur le programme Eumagine. L'un traite des discours et des images portant sur l'émigration marocaine ; en Europe, l'image du travailleur des années 1960-1970 reste la plus répandue (homme adulte et célibataire). Mohammed Adelghal analyse les arguments qui sont à l'origine des images négatives sur l'immigration : la menace 
que les immigrés représenteraient pour I'identité ethno-nationale ; la criminalité et I'insécurité dont ils seraient la cause ; le chômage qu'ils contribueraient à accroître ; I'attitude de profiteurs qui abuseraient de la générosité de l'État dans les démocraties occidentales. L'autre chapitre traite des images et des perceptions de l'Europe au sein de la société marocaine ; l'image du migrant marocain est souvent restreinte à celles du pourvoyeur de fonds. Mohamed Berriane montre la prédominance de l'Europe dans l'imaginaire marocain. La France est préférée aux autres pays d'Europe, car il y a du travail et des droits. À l'inverse, l'Espagne est perçue moins positivement du fait d'un nombre plus limité de droits et d'une crise économique plus prégnante. Enfin, l'image idyllique de l'Europe est fortement remise en question notamment à cause de l'insécurité et du développement du racisme. La question migratoire au Maroc a également trouvé à se diversifier depuis l'arrivée des Subsahariens. Ces nouvelles présences se sont accompagnées de commentaires médiatiques à connotation raciste quand des journaux ont pu choisir de titrer le " péril noir " pour décrire les mutations migratoires que connaît aujourd'hui le pays. Les opinions sont majoritairement négatives sur les services de santé et les écoles au Maroc, même si certaines sont plus équilibrées comme la liberté d'expression, la liberté linguistique ou l'égalité des chances par le travail entre hommes et femmes.

La deuxième partie de l'ouvrage traite des Marocains en Europe. Khayima Bouras Ostmann présente, dans le septième chapitre, les Marocains d'Allemagne. En 2010, les Marocains représentent $9 \%$ de la population étrangère en Allemagne (soit 151000 individus sur 7,5 millions de personnes). La plupart d'entre eux sont originaires du Rif. $50 \%$ des migrants marocains sont installés dans l'état de Rhénanie-du-Nord-Westphalie et $25 \%$ en Hesse. Depuis leur arrivée, ils ont surtout travaillé dans la mine, la sidérurgie, le bâtiment, l'industrie automobile et manufacturière. Aujourd'hui, le secteur tertiaire domine à 76 \% et le taux de chômage des Marocains a fortement augmenté.

Dans le huitième chapitre, Mathieu Rilke aborde l'arrivée des Marocains en Belgique dès 1912 et la convention bilatérale de 1964 qui marque l'afflux d'un grand nombre de travailleurs migrants. En 2011, 1119256 étrangers vivaient en Belgique, sur une population totale de 10951 266. Les Marocains représentent 7,4 \% avec 276587 personnes après les Français $(10 \%)$. La moitié d'entre eux vit dans la capitale belge, un tiers dans la Région flamande et le reste dans la Région wallonne. L'auteur démontre la faible intégration de cette population : les jeunes belgo-marocains connaissent des difficultés dans leur scolarité et sont touchés à $36 \%$ par le chômage (contre $7 \%$ en moyenne pour la population totale). Par contre, l'intégration politique a plutôt bien fonctionné grâce au droit de vote des étrangers mis en place dès 2006. La conclusion porte sur l'espoir que les BelgoMarocains ont pour leur descendance, car la mobilité sociale par l'éducation et le marché du travail sont beaucoup moins favorables qu'ils ne l'avaient prévu.

La situation des Marocains en Espagne est tout à fait différente, comme l'explique Mohammed Khaldi dans le chapitre 9. En 2008, avec la crise, la situation migratoire a complètement changé, inversant les flux. 1,2 million d'immigrés ont quitté l'Espagne entre 2008 et 2012 et les Espagnols commencent à émigrer à leur tour. Entre 2007 et 2010, le taux de chômage était de $35 \%$. Or l'immigration est principalement une immigration de travail et les travailleurs étrangers sont les plus touchés par la crise, notamment car ils occupaient des emplois non qualifiés dans le secteur du bâtiment qui a le plus souffert de la récession. L'Espagne est, pour les Marocains, le deuxième pays d'accueil après la France. Leur nombre est passé de 54 105, en 1992, à 854 501, en 2012, auquel 
s'ajoutent 120648 Marocains de nationalité espagnole, soit près d'un quart des Marocains dans le monde. Jusqu'en 2010, les Marocains sont la première communauté étrangère en Espagne. La population marocaine est jeune, avec une moyenne d'âge de vingt-huit ans (quarante-deux ans pour les Espagnols) et se concentre principalement dans trois régions : la Catalogne $(32,9 \%)$, l'Andalousie $(15,1 \%)$ et la région de Madrid (10,5\%). Près de la moitié $(49,3 \%)$ des Marocains en Espagne sont des demandeurs d'emploi.

En Europe, la France a constitué, pour des raisons historiques, le principal foyer de l'immigration marocaine, comme le mentionne Mohamed Charef dans le chapitre 10. Après un âge d'or de la migration marocaine " assistée " des années 1960, des milliers de Marocains sont venus travailler en France. De 1975 à nos jours, la population marocaine continue d'évoluer malgré un certain nombre de lois votées pour limiter la venue des étrangers. Le regroupement familial joue un rôle important dans l'essor de la population marocaine et dans sa féminisation. Cette population se naturalise également de plus en plus. Les principaux foyers de résidence sont le bassin Houiller du Nord-Pas-de-Calais et la région parisienne, devant les grandes zones maraîchères, arboricoles et viticoles (sud-ouest et sud-est), le secteur touristique du littoral méditerranéen et, bien sûr, les grandes villes. Enfin, l'auteur évoque les Marocains du " bout du Monde ", faisant allusion aux 4000 Marocains résidant dans les territoires d'outre-mer, dont la présence est relativement symbolique.

Le chapitre 11, développé par Immacolata Caruso et Sabrina Greco, analyse la venue importante des migrants marocains en Italie ces deux dernières décennies. Ils sont en 2011, 501 610, soit le plus fort contingent d'étrangers dans le pays $(14,2 \%)$. La plus grande concentration d'immigrés marocains $(73,8 \%)$ se localise dans les huit régions du Nord de I'Italie. Les villes de Turin, de Milan, Bergame, Brescia et Modène polarisent plus de $25 \%$ des immigrés marocains. Après la recherche d'un emploi, le regroupement familial occupe à présent une place importante dans les flux d'entrées en Italie. Mais depuis 2011, les ressortissants étrangers sont touchés par un chômage grandissant. Le taux de chômage au sein de la communauté marocaine est de $21 \%$. Parmi les actifs marocains, la moitié d'entre eux occupent des emplois dans le secteur du service. Entre le Maroc et I'Italie, il existe des accords bilatéraux concernant trois domaines : la gestion de la migration légale, la lutte contre l'immigration clandestine et enfin la promotion du lien entre migration et développement.

Les Marocains des Pays-Bas, étudiés par Mohammed Refass dans le chapitre 12, ont d'abord transité par la France ou la Belgique et sont ensuite venus plus directement du Maroc. Depuis 2009, ils se placent au deuxième rang des effectifs étrangers après les Turcs. Ils représentent 2,2 \% de la population néerlandaise totale, constituent plus du dixième de la population étrangère et plus de $18 \%$ de la population allochtone non occidentale des Pays-Bas. La région ouest concentre à elle seule près de $73 \%$ des Marocains. Amsterdam vient en tête des villes avec 19,84 \% de résidents d'origine marocaine pour un peu plus de $9 \%$ de la population totale de la ville. Rotterdam vient en seconde position. Avec la crise, le taux de chômage chez les Marocains est trois fois plus élevé que le taux moyen (19,6\% en 2012). Une politique d'immigration de plus en plus restrictive est mise en place avec l'encouragement au retour (projet REMPLOD). Les récents débats se sont focalisés sur la question identitaire et plus particulièrement autour de l'islam.

Dans le chapitre 13, Myriam Cherti appréhende les Marocains du Royaume-Uni, en expliquant tout d'abord les liens historiques tissés, dès le XIXe siècle, avec les marchands Fassis 
venus à Londres, Liverpool et Manchester. La migration marocaine en GrandeBretagne reste peu étudiée, car peu visible (elle regroupe 21246 personnes et place le groupe en 59ème position parmi les populations étrangères du Royaume), même si des espaces d'installation témoignent de cette présence comme à Londres avec le "le Petit Maroc " (quartier de North Kensigton). Un grand nombre de femmes sont arrivées en tant que migrantes indépendantes durant les années 1970, des célibataires, veuves, divorcées ou femmes cheffes de ménages. La première phase importante débute dans les années 1960 avec des Marocains (issus du Nord du Maroc) venant travailler dans le secteur de I'hôtellerie et de la restauration. Puis suivent de jeunes entrepreneurs semiqualifiés dans les années 1980 au départ de Casablanca. Enfin, dans les années 1990, des Marocains hautement qualifiés, provenant du Maroc et de France, arrivent pour travailler dans le secteur de la finance. La ville de Londres concentre $69 \%$ des Marocains. De manière générale, la communauté marocaine reste confrontée à de fortes difficultés liées à son manque de compétences linguistiques.

La troisième partie du livre porte sur les Marocains d'Amérique. Younes Abdelmoula traite du Canada dans le chapitre 14. Rajeunissement progressif, féminisation notable, niveau de scolarité et de qualification plus élevé sont les nouvelles tendances résultant de la politique migratoire canadienne, qui consiste à sélectionner et à choisir ceux qui ont les moyens de réussir leur intégration. C'est au début des années 1980 que s'accélère l'émigration marocaine. Estimée à 100000 personnes, elle est classée comme la première communauté maghrébine vivant au Canada, avec 8,1 \% de l'immigration canadienne totale entre 2001 et 2006. Pour des raisons linguistiques, $80 \%$ des Marocains s'établissent au Québec. $62 \%$ des actifs marocains sont concentrés dans des professions à haute qualification, $15 \%$ exercent des professions libérales ou sont cadres supérieurs. Toutefois, le chômage touche plus de $28 \%$ des Marocains du Canada $(8,3 \%$ pour le reste de la population immigrante).

Aux États-Unis, d'après Andrew A. Beveridge, Susan Weber et Sydney Beveridge dans le chapitre 15, avec 310 millions de personnes, la présence marocaine (66 352) actuelle est assez minime, concentrée dans les grandes villes telles que Los Angeles, San Francisco, Chicago, Boston, New York, Washington, etc. La zone de New York City compte plus d'un cinquième des Marocains des ÉtatsUnis. Pour résumer, la situation financière, professionnelle et éducative des Marocains est bien inférieure à la situation des autres groupes, excepté celui des autres pays d'Afrique. II y a cependant des différences notables entre les Marocains nés aux ÉtatsUnis et ceux nés à l'étranger.

Enfin, la dernière partie de l'ouvrage concerne les Marocains d'Afrique Subsaharienne et des pays arabes. Yahia Abou El Farah fait un zoom sur le Sénégal et la Côte d'Ivoire où sont plus fortement concentrés les Marocains. Le Sénégal a connu une immigration marocaine relativement ancienne au milieu du XIXe siècle, tandis que pour le second pays, elle est plus récente. Cette migration est due à la diffusion de I'Islam (surtout de la confrérie Tijania) et au développement du commerce caravanier d'où une communauté commerçante à Saint Louis issue de la grande " aristocratie marocaine Fassis ". L'émergence de la Côte d'Ivoire dans les années 1980 et 1990, comme nouvelle puissance économique d'Afrique avec l'exportation des produits agricoles (café, cacao, etc.) en fera une destination privilégiée. Par la suite, d'autres pays africains comme le Gabon, la Guinée, I'Afrique du Sud vont attirer des Marocains. En 2013, au Sénégal, les Marocains sont estimés à 2000 personnes et à 2700 en Côte d'Ivoire, auxquels il faut ajouter les 2 à 3000 métisses du Sénégal, les 800 étudiants, les femmes exerçant des activités liées 
aux attractions nocturnes, estimées entre 4 et 600 femmes, et quelques Marocains binationaux d'Europe. Avec la crise de 2011 en Côte d'Ivoire, la situation économique a impacté la communauté marocaine et a conduit une partie de la communauté marocaine à opter pour une migration de retour.

Pour Abdelfattah Ezzine, les migrations marocaines vers les pays arabes sont restées insignifiantes jusqu'aux années 1980. Si la migration marocaine vers l'Algérie reste la plus ancienne, celle vers le Liban a été aussi florissante sous l'occupation française. Avec le boom pétrolier, un nombre relativement limité de Marocains a migré vers la Libye (120 000) et les pays pétroliers du Golfe (plusieurs dizaines de milliers) pour travailler sous contrat temporaire surtout dans le secteur des services. Au début de ce siècle (2002), la communauté marocaine dans les pays arabes ne représentait que $8,99 \%$ des " Marocains du monde ». Dans l'aire arabe, le Maghreb et la Libye ont capté 59,86 \% des migrants marocains. Une des spécificités de cette migration dans les pays du Golfe est sa féminisation importante. En 2012, au Bahreïn, sur 1064 Marocains, $72,18 \%$ sont des femmes ; aux Émirats arabes unis, elles sont $60,2 \%$, à Oman, $65,5 \%$, au Koweït, 60,17 \%, en Syrie, $62,11 \%$, en Jordanie, 83,87 \% et en Arabie, seulement $42,4 \%$. La migration féminine est le résultat d'un projet migratoire individuel qui s'appuie sur les réseaux sociaux, familiaux, mafieux. Mais le système de la kafala la rend vulnérable.

En définitive, Mohamed Berriane, dans la coordination de cet ouvrage consacré aux Marocains de l'extérieur en 2013, nous apporte une lecture d'un paysage migratoire mondial des Marocains à un instant $\mathrm{T}$. Cet ouvrage est indispensable à tout chercheur, acteur de la société civile, migrant ou non migrant, Marocain ou non Marocain, voulant un peu mieux connaitre les Marocains du monde ou plutôt, le monde des Marocains, un monde en perpétuel mouvement. II est un outil pédagogique incontournable et attendu (environ tous les cinq ans) pour actualiser et mieux comprendre l'évolution d'une diaspora marocaine toujours plus nombreuse et qui compose un véritable miroir de la société marocaine du Maroc. Mieux connaître les Marocains de l'extérieur, c'est aussi mieux comprendre les Marocains du Maroc.

Chadia Arab

Chargée de recherche CNRS ESO/Université d'Angers

\section{Misrahi-Barak, Judith (dir.)}

Raynaud, Claudine (dir.)

Diasporas, Cultures of Mobility, "Race",

Vol. 1 Diasporas and Cultures of

Migrations. - Montpellier : Presses univer-

sitaires de la Méditerranée, 2014. - 376 p.

(PoCoPages)

ISBN : 978-2-36781-037-9

Diasporas, Cultures of Mobility, "Race", dirigé par Judith Misrahi-Barak et Claudine Raynaud, est le fruit de plusieurs rencontres ayant eu lieu en Europe et aux États-Unis entre 2011 et 2013. Cet ouvrage collectif rassemble des contributions d'auteurs principalement issus des études littéraires, mais également des sciences sociales, portant sur des études de cas précises. Ce recueil d'analyses est organisé en quatre parties : "Questioning diasporas ", " Diasporic Discontinuities ", "Diasporic Negotiations and Passages ", « Diasporic Subjects: Plural, Gendered, and Queer".

L'introduction annonce le principal enjeu théorique de l'ouvrage : repenser le concept de " diaspora ", notamment à partir de l'utilisation de sources littéraires et artistiques. Si le risque d'une dissolution de ce dernier est évoqué, en raison de la démultiplication de ses usages, les auteurs du recueil ne plaident néanmoins pas, dans leur majorité, pour son entière remise en cause. 
Comme Robin Cohen, beaucoup préfèrent aujourd'hui élargir le concept pour l'appliquer non plus seulement à des dispersions traumatiques, mais plus largement à des phénomènes migratoires se traduisant par l'extension de la " terre d'origine " au-delà de ses frontières, accompagnée d'une idéalisation de cette dernière et d'un fort sentiment " ethnique " et de solidarité avec les membres de la " diaspora ». Ainsi, I'article de Bénédicte Ledent analyse le roman In the falling snow (2009) de Caryl Phillips en proposant de penser autrement la diaspora : d'abord en termes de continuité et de discontinuité et également de travailler conjointement la singularité et la grande connexité des notions de migration, de diaspora et de globalisation. D'autres auteurs portent cependant plus loin la critique en rompant notamment avec la centralité accordée au rapport au " pays natal " dans la définition des phénomènes diasporiques. Ainsi, Shu-mei Shih, après avoir relevé les contradictions heuristiques et éthiques (réductionnisme ethnique, ethnocentrisme chinois, etc.) qui sous-tendent l'expression de " diaspora chinoise "-laquelle ne rend pas compte de l'hybridité et de l'hétérogénéité des logiques migratoires de ces populations - tente de poser les jalons d'un espace d'études sinophones. Le chapitre d' H. Adlai Murdoch portant sur l'identité caribéenne tend vers des visées similaires : ouvrir des analyses comparatives transdisciplinaires en réarticulant les concepts de différence, de multiplicité, de créolisation, d'hybridité, de métissage, etc. L'auteur mobilise la métaphore végétale du " rhizome " pour penser la mobilité dans toute sa complexité ce qui lui permet de contourner l'usage de celle des " racines " et l'idée d'origine commune qu'elle suggère. Ainsi, $H$. Adlai Murdoch plaide contre l'inscription trop réifiante d'un imaginaire du pays natal (au singulier) comme l'une des caractéristiques principales de la construction diasporique: la trame architecturale de l'identité culturelle diasporique est alors non plus soutenue par les motifs du retour mais de la différence.
D'autres contributions élargissent plus encore la portée du concept de " diaspora " qui se transforme alors en un outil facilitant la mise en lumière d'expériences entretenant a priori peu de rapport avec les phénomènes diasporiques à proprement parler. Ainsi J. U. Jacobs, dans une analyse de The Cry of Winnie Mandela de Njabulo Ndebele, dresse un parallèle entre l'expérience diasporique et la vie de plusieurs femmes sud-africaines touchées par la violence de leur pays : dans cette perspective, ces expériences ont pour point commun une forme de déstabilisation absolue dont rendrait compte la théorie du chaos. Plus largement que le concept de " diaspora " lui-même, c'est le champ sémantique qui l'accompagne qui est réexaminé. La contribution de Françoise Lionnet, par exemple, rejoignant les analyses $d^{\prime} H$. Adlai Murdoch et de Shu-mei Shih, alimente la réflexion autour des concepts de " créolité " et de " cosmopolitisme " ; concepts qui ont acquis une place importante aussi bien dans les études littéraires ou artistiques que dans les sciences sociales.

Refusant la lecture de la diaspora comme ensemble homogène, la plupart des auteurs mettent l'accent sur les discontinuités (Bénédicte Ledent, Mireille Rosello et J. U. Jacobs), I'hybridité (H. Adlai Murdoch) et les rapports de domination et les conflits en ce qu'ils pénètrent notamment les discours et les imaginaires diasporiques (Ashraf H. A. Rushdy, Louise Cainkar et Indira Karamcheti). L'expérience diasporique apparaît dans de nombreuses contributions comme suscitant ou accompagnant une remise en cause des normes sociales. Christine Vogt-William met par exemple en lumière le fait que les migrations des personnages principaux des romans de Shyam Selvadurai vont de pair avec l'affirmation progressive de masculinités alternatives en rupture avec I'hétéronormativité imposée dans leurs pays d'origine. Janet Wilson montre, quant à elle, comment l'expérience de l'exil de quatre écrivaines néo-zélandaises les 
conduit à questionner non seulement leur identité nationale ou " ethnique ", mais aussi leurs rôles de femmes. Ainsi, la mobilité semble favoriser le dévoilement de I'hétérogénéité des cultures et la difficulté d'en délimiter des contours clairement saisissables. Dans ce sens, I'analyse du film Gadjo Dilo par Mireille Rosello peut être lue comme une contribution à la réflexion postmoderne sur le caractère " ouvert ", hétérogène et conflictuel des cultures et des normes sociales.

La dialectique continuité/discontinuité n'est pas uniquement posée en référence à un cadre social ou géographique, mais également temporel. En effet, la nécessité d'une perspective diachronique pour saisir les logiques et l'évolution du processus diasporique et des identités infuse nombre de chapitres de l'ouvrage. C'est le cas de celui de Bénédicte Ledent, mais aussi de Shanthini Pillai qui s'intéresse aux négociations de la mémoire et de l'espace à travers deux romans d'écrivains de la diaspora malaise (Preeta Samarasan et Hsu Ming Teo). De même, Corinne Duboin, à travers l'étude de trois fictions de Dinaw Mangestu et Chimamanda Ngozi Adichie, analyse les variations avec lesquelles ces auteurs, considérés comme appartenant à la " seconde génération " d'écrivains africains aux États-Unis nés à l'étranger, abordent les problématiques relatives à l'appartenance, aux identités et aux filiations. Leurs travaux soulignent notamment l'importance d'une lecture générationnelle, genrée, incluant affects et émotions, rendue possible par l'analyse des textes littéraires. Avec une approche différente, le sociolinguiste Lars Hinrichs prend lui aussi en compte la question générationnelle et montre que, parmi les migrants jamaïcains de seconde génération vivant à Toronto, la rupture n'est peut-être pas tant entre ceux nés en Jamaïque et les autres, mais entre des univers sociaux valorisant l'accent jamaïcain et d'autres ayant intériorisé sa stigmatisation.
Hormis de rares exceptions, les contributions reposent principalement sur l'utilisation de la littérature ou d'arts visuels. L'un des intérêts de l'ouvrage vient du fait que ces œuvres sont traitées non pas seulement comme un miroir du social, mais qu'elles sont aussi perçues comme irremplaçables pour comprendre les imaginaires et les affects au cœur des processus de construction des diasporas. Ce traitement des œuvres contribue à nourrir l'effort de questionnement et de conceptualisation (ou reconceptualisation) des postulats théoriques.

Le défi serait alors d'appréhender des œuvres littéraires ou artistiques comme ayant un statut épistémologique équivalent, voire supérieur, à celui de matériaux produits par l'enquêteur en sciences sociales. Les implications et les questions que ce point de vue soulève sont finalement peu abordées et discutées, si ce n'est dans la contribution de Janet Wilson ou dans celle de Bénédicte Ledent. Le seul regret que l'on puisse éprouver à la lecture de cet ouvrage, singulièrement stimulant, vient de la trop faible prise en compte de ces considérations, celle-ci rendant, in fine, le dialogue entre études littéraires et sciences sociales assez limité - ces dernières étant d'ailleurs peu représentées. Malgré cela, l'effort de la plupart des contributeurs littéraires pour inscrire leurs travaux dans une perspective transdisciplinaire demeure très convaincant.

Aurélie Condevaux

Chargée de cours

Université François Rabelais de Tours

Mélanie Pénicaud

Doctorante

MIGRINTER/Université de Poitiers 
Bredeloup, Sylvie

Migrations d'aventures. Terrains africains. Paris : CTHS Géographie, 2014. - 143 p.

ISBN : 978-27355-0818-1

À quel univers de sens se réfère le vocabulaire de la recherche dès lors qu'il relève d'un usage commun ? C'est à la construction sémantique de la notion $\mathrm{d}^{\prime}$ " aventure " et à la déclinaison de ses multiples enjeux dans le champ des migrations africaines que s'emploie Sylvie Bredeloup dans ce stimulant ouvrage. Socio-anthropologue de formation et spécialiste des migrations internationales, l'auteure s'est attelée à ce chantier en puisant dans une vaste culture scientifique et littéraire les nombreuses références permettant de mettre en évidence les vertus heuristiques du terme tout en questionnant les impensés qui l'entourent. Revisitant l'historiographie des migrations africaines depuis les années 1970, elle montre combien l'aventure constitue un angle de vue original et pertinent pour souligner l'inventivité, la créativité, mais aussi la prise de risques consentie qu'effectuent les migrants à différentes étapes de leurs parcours migratoires. Ce faisant, l'auteure pose un nouveau cadre d'analyse des migrations africaines qui s'affranchit des problématiques liées au co-développement ou orientées vers la recherche des déterminants économiques tout en proposant un certain nombre de pistes à investiguer destinées à complexifier ces approches.

Si l'ouvrage s'inscrit dans le prolongement de recherches portant sur la créativité de l'agir dans le domaine des migrations, domaine marqué par l'imprévu et l'inattendu, il se caractérise aussi par un travail de construction théorique de l'aventure comme catégorie d'analyse. Pour ce faire, I'auteure mobilise une approche généalogique permettant de relever les normes et les significations qui balisent l'espace de références et spécifient le mot depuis ses premières occurrences au XVle siècle sous la plume d'un chroniqueur (p. 19) et dans une discipline telle que la géographie, en 1975 (p. 10). La polysémie du terme, et les multiples sens auxquels il renvoie selon les contextes spatio-temporels, sont analysés comme autant de révélateurs des représentations qui parcourent différentes sociétés à différentes époques.

Pour autant, dans un contexte où l'inconnu a laissé place au connu et où il devient de plus en plus difficile pour les candidats à l'émigration de partir, l'usage actuel du terme aventure et sa prégnance dans le paysage social et médiatique ne peut qu'attiser la curiosité du chercheur. Quelles références s'approprie l'individu quand il s'auto-désigne ou désigne ses activités comme relevant de l'aventure ? Quelle est la valeur heuristique du terme pour penser autrement les mobiles de l'action ? Sylvie Bredeloup montre ainsi combien le recours à l'aventure témoigne de la progressive montée en puissance de la "réalisation de soi " comme dimension de l'action. Les terrains africains investigués depuis ses recherches sur les marchands de diamants jusqu'aux différentes générations de marins et d'entrepreneurs africains à Marseille témoignent de l'importance de ce registre dans le déclenchement de projets migratoires et dans le regard porté par les migrants pour qualifier leurs mobilités au regard des épreuves endurées. Dans cette perspective, le migrant est celui ou celle qui considère son parcours comme étant jalonné de défis à surmonter. II en résulte une conception de la migration envisagée comme une ouverture à l'imprévu et un moyen privilégié pour s'affranchir d'un destin connu d'avance.

Les neuf chapitres qui composent l'ouvrage apparaissent comme autant de déclinaisons permettant de creuser les multiples dimensions auxquelles renvoie l'aventure. Éloigné de tout académisme, mais au risque à certains moments de prendre au dépourvu le lecteur peu au fait du sujet par l'abondance des références puisées dans différents domaines, l'objectif 
n'est pas seulement de faire le point sur la notion - au sens parfois photographique du terme -, il est également de montrer comment elle renouvelle l'analyse des formes culturelles de la migration (p. 17).

Le premier chapitre déploie l'interrogation sur le sens donné à l'aventure dans le contexte européen. L'auteure met ainsi en avant la quête d'intensité comme ce qui différencie l'aventurier du héros, cette autre figure productrice de modèles d'identification. Les deux chapitres suivants poursuivent le travail de spécification de la notion en montrant comment la quête d'aventure n'est pas l'apanage du migrant, le sédentaire peut être également animé par cette recherche. Elle souligne aussi combien il serait réducteur de considérer toutes les migrations à travers ce prisme. Après ces éléments de clarification, l'auteure s'attèle à montrer, dans les chapitres suivants, comment des figures majeures de la migration africaine - le " jaguar " et le " sapeur " - peuvent constituer des modèles choisis par un grand nombre de jeunes pour le potentiel d'aventures auquel ils sont associés (chapitre 4). Un questionnement plus ample se dégage ainsi sur la dimension individuelle ou collective de l'aventure et sur les modes de promotion sociale qu'elle rend possible. Si s'éloigner du groupe est la condition nécessaire à la réalisation de soi pour nombre d'aventuriers, la reconnaissance du caractère aventureux de la démarche s'effectue par le groupe. Ainsi, la notion apparaît-elle comme un révélateur de la façon dont se pense le changement social au sein des collectifs et de la façon dont il est perçu (chapitre 5). L'intensité du vécu qui caractérise l'aventure peut-elle cependant se manifester sur toute une vie ? Le chapitre 6 aborde cette question en soulignant l'importance des actions ritualisées qui rythment la vie d'un collectif à travers le devenir des siens. L'auteure présente également les principales voies de sortie de l'aventure, ce qui consacre I'hypothèse selon laquelle l'aventure ne peut être que ponctuelle, mais pas nécessairement vécue comme telle par les migrants rencontrés. Les territoires de I'aventure font l'objet du chapitre suivant. Si chaque époque a eu ses territoires de déploiement, la grande ville se présente désormais comme un lieu majeur d'expérimentation ; l'aventure se cristallise en effet et affleure dans une toponymie urbaine officieuse, mais révélatrice des nombreux chemins détournés empruntés par celles et ceux qui souhaitent se distinguer par leur capacité à innover. Le chapitre 9, consacré aux imaginaires migratoires, oriente la réflexion vers l'analyse des opérations de (re)qualification qu'effectuent les migrants à différentes étapes de la mise en récit de leurs parcours. Le rôle et la place des femmes migrantes ainsi que les possibilités qui s'offrent à elles pour sortir des sentiers balisés font l'objet du dernier chapitre, lequel précède une riche conclusion récapitulative.

Au final, l'ouvrage pose les bases d'un programme de recherche innovant sur l'aventure comme prisme opératoire dans la mise en valeur d'aspects souvent minorés ou occultés, mais décisifs dans la réalisation des parcours migratoires. Si les terrains investigués sont africains, ce programme a cependant vocation à s'étendre à d'autres aires géographiques afin de comprendre comment la quête d'aventure se présente comme un mobile important dans l'activation de parcours de mobilité à la fois géographique et sociale. L'ouvrage apporte ainsi une contribution significative au paradigme de l'autonomie dans les mobilités et migrations internationales. II intéressera, à ce titre, un public d'universitaires pour l'actualité vive de la recherche sur le sujet et un grand public désireux de porter un regard affranchi de considérations misérabilistes sur les migrations d'aventure qui se déploient dans le berceau de l'humanité.

Constance De Gourcy

Enseignante-chercheure, Département de sociologie Aix-Marseille Université/LAMES 
Tarrius, Alain

Bernet, Olivier

Mondialisation criminelle. La frontière

franco-espagnole de La Junquera à

Perpignan. Rapport de Recherche. - Saint-

Denis, Edilivres, 2014. - 109 p.

ISBN : 978-2-332-74453-1

Tout au long de sa carrière, Alain Tarrius, professeur émérite de sociologie à I'Université Toulouse 2-Le Mirail, n'a eu cesse de lever le voile sur les faits sociaux les moins visibles, de gratter patiemment le vernis de nos sociétés pour donner à voir et à comprendre ce qui se joue dans les interstices, mais qui relève de logiques plus vastes. Force est de constater qu'il n'en a pas fini et qu'avec la même pugnacité, il livre " à chaud ", avec le sociologue Olivier Bernet, le dernier numéro de la collection Recherches en cours/cosmopolitismes méditerranéens, chez l'éditeur en ligne Edilivres. À partir d'une étude de la frontière franco-espagnole à La Junquera soutenue par le Labex SMS, Université de Toulouse, les auteurs suivent deux pistes. La première retrace les parcours de la prostitution et de la drogue de la mer Noire et des Balkans jusqu'au Levant espagnol. La seconde, imprévue, suivie par l'intuition des chercheurs, s'attache à montrer que les réseaux de la mondialisation criminelle présents du côté espagnol de la frontière ont trouvé un terreau favorable dans le département frontalier des PyrénéesOrientales.

\section{De la mer Noire au Levant espagnol}

Les transmigrations sont toujours dans la focale d'Alain Tarrius et d'Olivier Bernet. La première partie de la publication est consacrée aux parcours prostitutionnels de femmes originaires des pourtours de la mer Noire ou des Balkans, accompagnées par les réseaux criminels qui les encadrent et par la circulation des drogues. À partir des entretiens menés avec quelques-unes de ces femmes, et dont le verbatim très présent ajoute encore à l'implication du lecteur, les auteurs retracent les parcours, les étapes sordides et les issues inégales. Le parcours de Magdalena, Irina et Sofia commence sur les paquebots et cargos qui sillonnent cette mer. Le stage à bord ou l'escale au port, à Sotchi - ville olympique-, Trabzon ou Odessa, deviennent les lieux des premières passes. Le repérage ne tarde pas. Marins à bord ou logeurs de retour au pays, les recruteurs agissent pour le compte d'un large réseau diffusant femmes et drogues vers les Émirats, le Levant espagnol, I'Italie ou laTurquie.

La prise en charge de ces femmes par les réseaux criminels des pourtours de la mer Noire est également une étape de triage. Les " meilleures " sont directement cédées à des acquéreurs des Émirats ou d'Espagne. Irina et sa sœur, déshabillées, palpées, essayées par leurs acheteurs lors d'une vente aux enchères, sont vendues pour 5000 euros chacune et envoyées en Catalogne après une rapide formation de dealer. Car la vente des drogues est intimement associée à celle des corps. La marchandisation est totale. D'autres, comme Magdalena, doivent passer par une étape de rattrapage, en Italie, dans la région de Salerne. Les réseaux, contrôlés notamment par des Géorgiens, fournissent les papiers nécessaires au voyage et la drogue une fois en Espagne. Régularisées, travaillant dans les clubs luxueux du Levant espagnol, certaines peuvent alors prendre un peu mieux le contrôle de leur parcours et établissent une véritable stratégie de voyage, organisant peu à peu leur retour comme c'est le cas d'Irina et de Sofia.

Parmi les parcours évoqués, il y a aussi ceux des déclassées à l'image de Sardinella. Albanaise catholique envoyée pour son " noviciat " àTarente, elle s'enfuit et, "de ratage en ratage ", comme elle le dit, tombe amoureuse d'un homme qui la prostitue dans la cale du bateau de pêche de son patron, lui-même en affaire avec la mafia locale. Privée de toute initiative, elle est embarquée par les mafieux après l'élimination de ses proxénètes. Débarquée au port de La Escala, elle est finalement cédée 
à un abattoir catalan, non pour elle-même, mais comme prime d'une transaction de stupéfiants. II y a bien quelque chose ici de la misère montrée dans Gomorra, célèbre roman de Roberto Saviano.

\section{La " moral area "}

Les deux sociologues s'appuient largement sur la notion de " moral area " ou espace de mœurs, proposée par le sociologue Robert Ezra Park, I'un des fondateurs de l'École de Chicago dans les années 1920. Elle leur permet de comprendre les continuités spatiales cachées des économies de l'argent et du désir'1.

Si la mer Noire est sans conteste une " moral area " dans la première partie du propos, c'est aussi le cas de la Catalogne qui semble retrouver son unité dans les économies peu visibles du sexe et de la drogue, de Perpignan à la station balnéaire " branchée " de Sitges, au sud de Barcelone. Les auteurs montrent que les réseaux mafieux, les centralités de prostitution masculine (Sitges) ou féminine (La Junquera), les camionneurs, les bourgeoisies locales, participent à cette zone de mœurs et sont parties prenantes d'un dispositif qu'il ne faudrait pas confondre avec ses manifestations visibles que sont les espaces de prostitution.

À partir de l'étude de la centralité criminelle et frontalière de La Junquera, débouché des réseaux de drogue et de prostitution russo-italiens, les auteurs

\footnotetext{
1 La notion de " moral area ", ou " zone de mœurs ", est utilisée par l'ensemble des sociologues de l'École de Chicago pour décrire l'attraction et le brassage, souvent nocturne et dérogatoire de "l'ordre urbain ", de populations variées autour d'activités mêlant plaisirs et intérêts. Cf Hannerz UIf (1983) Explorer la ville, Paris, Éditions de Minuit, 432 p. Les " moral area " jouent un rôle essentiel, bien que non reconnu, masqué, dans les transformations urbaines. Une autre traduction de " moral area " par " district moral " est proposée par Isaac Joseph.
}

s'aperçoivent que le département voisin des Pyrénées-Orientales a aussi trouvé sa place dans le dispositif criminel. C'est un espace annexe de vente des drogues. Les puissants réseaux qui opèrent à $\mathrm{La}$ Junquera acquièrent rapidement le monopole des drogues les plus lucratives au détriment des petits distributeurs locaux. Pour maintenir leurs bénéfices, ces derniers élargissent leur clientèle en vendant des drogues chimiques aux collégiens et lycéens de ce département.

C'est en conduisant ce volet de l'enquête, et notamment en cherchant à signaler la situation de jeunes pourtant placés par l'Aide sociale à l'enfance départementale (ASE 66) que les chercheurs mettent progressivement à jour un système clientélique local. En effet, il semblerait que certains enfants soient placés dans des familles d'accueil, elles-mêmes en situation de misère sociale, comme attribution d'un revenu. La volonté de conserver ce revenu justifierait de passer sous silence tout dysfonctionnement (drogue, prostitution) à même de provoquer un retrait de I'enfant de sa famille d'accueil. Les auteurs s'attachent ensuite à exposer la virulence de l'institution à se défendre de l'intrusion des chercheurs, livrant quelques pages offensives, parfaitement assumées, qui restent encore trop rares dans les sciences sociales.

À partir de ces blocages, les chercheurs développent leurs enquêtes auprès des jeunes prostitués et drogués de la région de Perpignan qui leur permettent de confirmer l'intégration des PyrénéesOrientales à la " moral area " sud-catalane des drogues et de la prostitution. Notamment quand, et c'est à peine un raccourci, de jeunes Perpignanais, certains encore placés par l'ASE 66, sous amphétamines géorgiennes, partent pour la saison estivale se prostituer dans les clubs de Sitges. 


\section{Des chercheurs inlassables et inclassables}

Cet ouvrage, qui devrait donner lieu à d'autres enquêtes et d'autres publications, est une sorte de rapport d'étape d'une recherche collective. Le propos est soutenu par un dispositif de recherche complexe, dépassant la personnalité des deux auteurs. Pourtant, c'est bien Alain Tarrius qui peut, avec une virulence déroutante puis communicative, donner le " coup de pied dans la fourmilière ". À I'instar de Pierre Bourdieu, il considère que la sociologie est " un sport de combat " et le démontre énergiquement. Quand une porte se ferme, il s'agit davantage d'une injonction à aller voir - sans voyeurisme - ce qui se cache derrière, à ouvrir un nouveau volet, imprévu, de l'enquête.

Ici, les chercheurs sont engagés et le propos, toujours étayé, est bien entendu scientifique. Mais le texte est inclassable et peu conventionnel, tant par sa forme que par le ton combatif qu'il emploie parfois, pour le plus grand bien du lecteur profitant d'un contenu informatif riche et assistant au déploiement d'une pratique de recherche engagée. Enfin, les annexes étoffées présentent un corpus de textes et $d$ 'articles publiés par les auteurs et formant une sorte de précis théorique et méthodologique (paradigme de la mobilité, territoires circulatoires) autour du concept de transmigration.

Adrien Doron

Doctorant en géographie Université Toulouse Jean Jaurès/LISST
Berthès, Colette

L'exil et les barbelés. - Paris, Riveneuve, 2011. $-225 \mathrm{p}$.

ISBN : 978-2-360-13038-2

Malgré son titre, L'exil et les barbelés n'est pas un essai sur l'exil confronté aux barbelés, mais un excellent documentaire historique sur le camp de concentration (dénomination officielle) de Septfonds, en Tarn-et-Garonne, construit en 1939 pour interner 15000 réfugiés républicains espagnols, utilisé par le régime de Vichy pour y enfermer des juifs et des opposants, réutilisé à la libération pour emprisonner des personnes accusées de collaborations avec l'ennemi, et finalement détruit en 1945.

L'auteur retrace l'histoire du camp, de ses occupants successifs et de sa perception par la population locale à partir d'un important travail de recherche documentaire : archives officielles et privées, journaux d'époque, ouvrages divers et entretiens avec des personnes ou leurs descendants qui ont connu le camp. Ce recueil d'informations est d'autant plus important que certaines archives des diverses administrations du camp ont été brûlées en 1945 avec l'accord du préfet.

Les très nombreux détails sur le camp et les petites citations d'écrits des acteurs de l'époque rendent cet ouvrage très vivant et agréable à lire. On peut néanmoins regretter quelques répétitions liées au plan adopté qui superpose un récit chronologique, un récit par thème sur la vie des exilés au camp (la maladie et la mort, manger boire dormir, tuer l'ennui, les fêtes et cérémonies, etc.) et un récit par thème sur la vie du camp lui-même (la construction du camp, le camp de Septfonds mars 1940-mai 1945, rafles et déportation des juifs, le temps de l'épuration, etc.).

Bien que l'auteure ne prétende pas à une totale objectivité, elle évite les jugements de valeur sur les différents acteurs, tout en nous montrant que les 
soldats de l'armée républicaine espagnole " sont traités non comme des soldats vaincus réfugiés en pays ami, mais comme des soldats prisonniers d'autant plus dangereux que les officiers du camp les considèrent tous comme "communistes" ".

Le grand mérite de cet ouvrage est de décrire très concrètement la réalité peu connue d'un camp de concentration français dans cette période troublée.

Luc Legoux

Démographe

IDUP/Université Paris I

Hanus, Philippe

Teulières, Laure

Vercors des mille chemins. Figures

de l'étranger en temps de guerre. -

Rochechinard, Comptoir d'éditions, 2013. $319 \mathrm{p}$.

ISBN : 978-2-919163-03-8

Une trentaine d'auteurs se sont réunis pour étudier le phénomène migratoire dans les Vercors et les régions proches, cela durant la Deuxième Guerre mondiale, avec des regards sur la période précédente et la période suivante, ce qui permet d'évoquer la mémoire des années 19391945. Les auteurs ont puisé leurs informations dans les archives, les témoignages oraux, les récits de vie dont certains sont reproduits in extenso.

Pour la période antérieure à la guerre, l'immigration économique est présentée grâce à une monographie portant sur les employés étrangers travaillant dans I'hôtellerie à Villard-de-Lans comme simples salariés ou dirigeants d'établissements parfois prestigieux. L'immigration politique est vue à travers le prisme de l'antifascisme italien dans I'lsère, communiste et non-communiste. Le tableau est complété par des études sur les réfugiés espagnols du Royans et du Vercors, leurs relations cordiales ou conflictuelles avec la popula- tion locale, le contrôle policier de ces hôtes très politisés.

Les pages consacrées à l'occupation s'ouvrent par une recherche sur les Italiens dans l'espace alpin, la diversité professionnelle de cette population, la méfiance qu'elle éveilla, les départs encouragés par Mussolini, le régime de faveur que permit I'appartenance de l'Italie au camp des vainqueurs, la désertion de nombreux soldats transalpins après juillet 1943. Les autres analyses sont consacrées aux enfants juifs accueillis à Izieu et au village des Justes de Prélenfrey dont aucun habitant ne dénonça la présence des jeunes, présentés tous comme tuberculeux alors que seul un tiers était affecté par la maladie. Le centre d'accueil de Pont-de-Manne hébergeait des adultes juifs libérés des camps et pris en charge par l'infatigable abbé Glasberg. De 1940 à 1946, quelque 700 jeunes fréquentèrent le lycée polonais de Villars-de-Lans. Quelques AngloSaxons généralement âgés passèrent la guerre dans des hôtels de la région. Le Vercors reçut aussi des bûcherons indochinois, vivant dans des conditions très précaires, ce qui n'empêcha pas certains de s'engager dans la Résistance. Dans les rangs de celle-ci se retrouvèrent aussi des antifascistes allemands et autrichiens. Le Polonais Marco Lipszyc commanda les Francs-Tireurs Partisans de I'Isère. Des tirailleurs sénégalais participèrent à la bataille du Vercors en 1944 et à la libération de Romans.

Après la guerre, des rescapés étrangers revinrent en visite dans la région, écrivirent aux Français qu'ils avaient alors connus et les invitèrent parfois dans leur pays d'origine. Les artistes locaux s'inspirèrent des événements vécus entre 1939 et 1945. La vie reprit son cours et les anciens courants d'immigration tendirent à se remettre en place, notamment grâce au recrutement d'Italiens employés au relèvement des ruines. 
Les textes qui figurent dans Vercors des mille chemins se révèlent d'un intérêt inégal. Mais, juxtaposés, ils forment une sorte de kaléidoscope qui restitue bien la diversité des étrangers ayant résidé dans la région, touristes avant la guerre, travailleurs immigrés, réfugiés politiques, Européens, Africains, Indochinois, etc. Le parcours personnel de tous ces hommes, leurs expériences, leurs épreuves sont conformes à ce qui fut observé dans d'autres régions françaises. Les étrangers furent confrontés, selon les cas, à la méfiance et à I'hostilité ou, au contraire, entourés par une chaude solidarité et des gestes d'amitié. II semble qu'au total les attitudes positives l'emportèrent et que, de part et d'autre, bien des préjugés s'effacèrent. Petite consolation pour une période de drames.

Ralph Schor

Professeur émérite d'histoire Université de Nice-Sophia-Antipolis 